\title{
On finite pseudorandom binary sequences VII: The measures of pseudorandomness
}

\author{
by \\ Julien Cassaigne (Marseille), Christian Mauduit (Marseille) \\ and ANDRÁs SÁRKÖZY (Budapest)
}

1. Introduction. In this series we study finite pseudorandom binary sequences $E_{N}=\left\{e_{1}, \ldots, e_{N}\right\} \in\{-1,+1\}^{N}$. In particular, in Part I [MSá] we introduced the following measures of pseudorandomness: Write

$$
U\left(E_{N}, t, a, b\right)=\sum_{j=0}^{t-1} e_{a+j b}
$$

and, for $D=\left(d_{1}, \ldots, d_{k}\right)$ with non-negative integers $0 \leq d_{1}<\ldots<d_{k}$,

$$
V\left(E_{N}, M, D\right)=\sum_{n=1}^{M} e_{n+d_{1}} e_{n+d_{2}} \ldots e_{n+d_{k}} .
$$

Then the well-distribution measure of $E_{N}$ is defined as

$$
W\left(E_{N}\right)=\max _{a, b, t}\left|U\left(E_{N}, t, a, b\right)\right|=\max _{a, b, t}\left|\sum_{j=0}^{t-1} e_{a+j b}\right|
$$

where the maximum is taken over all $a, b, t$ such that $a, b, t \in \mathbb{N}$ and $1 \leq a \leq$ $a+(t-1) b \leq N$, while the correlation measure of order $k$ of $E_{N}$ is defined as

$$
C_{k}\left(E_{N}\right)=\max _{M, D}\left|V\left(E_{N}, M, D\right)\right|=\max _{M, D}\left|\sum_{n=1}^{M} e_{n+d_{1}} e_{n+d_{2}} \ldots e_{n+d_{k}}\right|
$$

where the maximum is taken over all $D=\left(d_{1}, \ldots, d_{k}\right)$ and $M$ such that $M+d_{k} \leq N$.

2000 Mathematics Subject Classification: Primary 11K45.

Key words and phrases: pseudorandom, binary sequence, correlation.

Research of András Sárközy partially supported by the Hungarian National Foundation for Scientific Research, Grant No. T029759, MKM fund FKFP-0139/1997 and French-Hungarian APAPE-OMFB exchange program F-5/97. This paper was written while he was visiting the Institut de Mathématiques de Luminy. 
In Part I first we discussed several elementary properties of these pseudorandom (briefly: PR) measures. In the second half of Part I and in Parts II-VI, we tested special sequences for pseudorandomness. This testing was based on the principle formulated in [CFMRS1] in the following way: "The sequence $E_{N}$ " is considered as a "good" PR sequence if these measures $W\left(E_{N}\right)$ and $C_{k}\left(E_{N}\right)$ (at least for "small" $k$ ) are "small".

In this paper our goal is to return to the analysis of the general properties of these PR measures. First in Section 2 we will show that the testing principle quoted above is justified and, indeed, for a truly random $E_{N} \in\{-1,+1\}^{N}$ both PR measures $W\left(E_{N}\right)$ and $C_{k}\left(E_{N}\right)$ are "small". These results inspire a further question which, although less important from a practical point of view, seems to be of independent interest: for fixed $N, k$, what is the minimum of $W\left(E_{N}\right)$ and $C_{k}\left(E_{N}\right)$ ? This problem will be studied in Section 3. Finally, one might like to know whether it suffices to study correlation of order, say, 2, or correlations of higher order must be studied as well. This question can be answered by analyzing the connection between $C_{k}\left(E_{N}\right)$ and $C_{l}\left(E_{N}\right)$ for $k \neq l$; this analysis will be carried out in Section 4.

2. The PR measures for random binary sequences. In this section we will estimate $W\left(E_{N}\right)$ and $C_{k}\left(E_{N}\right)$ for "random" binary sequences $E_{N} \in\{-1,+1\}^{N}$, i.e., for choosing each $E_{N} \in\{-1,+1\}^{N}$ with probability $1 / 2^{N}$. We will show that for a random $E_{N}$ both $W\left(E_{N}\right)$ and $C_{k}\left(E_{N}\right)$ are around $\sqrt{N}$ :

Theorem 1. For all $\varepsilon>0$ there are numbers $N_{0}=N_{0}(\varepsilon)$ and $\delta=\delta(\varepsilon)$ such that for $N>N_{0}$ we have

$$
\begin{aligned}
& P\left(W\left(E_{N}\right)>\delta N^{1 / 2}\right)>1-\varepsilon, \\
& P\left(W\left(E_{N}\right)>6(N \log N)^{1 / 2}\right)<\varepsilon .
\end{aligned}
$$

Theorem 2. For all $k \in \mathbb{N}, k \geq 2$ and $\varepsilon>0$ there are numbers $N_{0}=$ $N_{0}(\varepsilon, k)$ and $\delta=\delta(\varepsilon, k)$ such that for $N>N_{0}$ we have

$$
\begin{aligned}
& P\left(C_{k}\left(E_{N}\right)>\delta N^{1 / 2}\right)>1-\varepsilon, \\
& P\left(C_{k}\left(E_{N}\right)>5(k N \log N)^{1 / 2}\right)<\varepsilon .
\end{aligned}
$$

Thus with probability $>1-2 \varepsilon$ we have

$$
\begin{aligned}
& \delta N^{1 / 2}<W\left(E_{N}\right)<6(N \log N)^{1 / 2}, \\
& \delta N^{1 / 2}<C_{k}\left(E_{N}\right)<5(k N \log N)^{1 / 2} .
\end{aligned}
$$

(2.5) could be improved with some effort but we did not force this since it is good enough for our purpose in this easier form. On the other hand, it seems to be more difficult to improve on (2.6). 
Proof of Theorem 1. First we will prove (2.1). Since by (1.1),

$$
W\left(E_{N}\right) \geq\left|U\left(E_{N}, N, 1,1\right)\right|=\left|\sum_{j=1}^{N} e_{j}\right|,
$$

we have

$$
P\left(W\left(E_{N}\right)>\delta N^{1 / 2}\right) \geq P\left(\left|\sum_{j=1}^{N} e_{j}\right|>\delta N^{1 / 2}\right),
$$

so that it suffices to prove

$$
P\left(\left|\sum_{j=1}^{N} e_{j}\right|>\delta N^{1 / 2}\right)>1-\varepsilon .
$$

If

$$
\left|\left\{j: 1 \leq j \leq N, e_{j}=-1\right\}\right|=h,
$$

then

$$
\begin{aligned}
\sum_{j=1}^{N} e_{j} & =\left|\left\{j: 1 \leq j \leq N, e_{j}=+1\right\}\right|-\left|\left\{j: 1 \leq j \leq N, e_{j}=-1\right\}\right| \\
& =(N-h)-h=N-2 h .
\end{aligned}
$$

(2.8) holds with probability $\frac{1}{2^{N}}\left(\begin{array}{l}N \\ h\end{array}\right)$, so that

$$
\begin{aligned}
P\left(\left|\sum_{j=1}^{N} e_{j}\right|>\delta N^{1 / 2}\right) & =\sum_{h:|N-2 h|>\delta N^{1 / 2}} \frac{1}{2^{N}}\left(\begin{array}{c}
N \\
h
\end{array}\right) \\
& =\frac{1}{2^{N}} \sum_{h:|h-N / 2|>(\delta / 2) N^{1 / 2}}\left(\begin{array}{c}
N \\
h
\end{array}\right) .
\end{aligned}
$$

It is a well known property of the binomial distribution that for all $\varepsilon>0$ there is an $\eta=\eta(\varepsilon)>0$ such that

$$
\sum_{h:|h-N / 2|>\eta N^{1 / 2}}\left(\begin{array}{c}
N \\
h
\end{array}\right)>(1-\varepsilon) 2^{N} .
$$

If we now choose $\delta=2 \eta(\varepsilon)$, then (2.7) follows from (2.9) and (2.10), and this completes the proof of (2.1).

Now we prove $(2.2)$. Write $L=6(N \log N)^{1 / 2}$. By (1.1) we have

$$
\begin{aligned}
P\left(W\left(E_{N}\right)>L\right) & =P\left(\max _{a, b, t}\left|U\left(E_{N}, t, a, b\right)\right|>L\right) \\
& \leq \sum_{a, b, t} P\left(\left|U\left(E_{N}, t, a, b\right)\right|>L\right)
\end{aligned}
$$


where both the maximum and the summation are taken over all $a, b, t \in \mathbb{N}$ such that

$$
1 \leq a \leq a+(t-1) b \leq N
$$

It follows that

$$
a, b, t \in\{1, \ldots, N\} .
$$

By (2.11) and (2.13) we have

$$
P\left(W\left(E_{N}\right)>L\right) \leq N^{3} \max _{a, b, t} P\left(\left|U\left(E_{N}, t, a, b\right)\right|>L\right)
$$

where again the maximum is taken over all $a, b, t$ satisfying (2.12). Thus in order to prove (2.2), it suffices to show that for all $a, b, t$ satisfying (2.12),

$$
P\left(\left|U\left(E_{N}, t, a, b\right)\right|>L\right)=P\left(\left|\sum_{j=0}^{t-1} e_{a+j b}\right|>L\right)<\varepsilon / N^{3} .
$$

If $t \leq L$ then the probability in (2.14) is trivially 0 so that we may assume that

$$
t>L=6(N \log N)^{1 / 2}
$$

Write

$$
\begin{gathered}
M=6(t \log t)^{1 / 2}, \\
\left|\left\{j: 0 \leq j \leq t-1, e_{a+j b}=-1\right\}\right|=h .
\end{gathered}
$$

Then

$$
\begin{aligned}
\sum_{j=0}^{t-1} e_{a+j b}= & \left|\left\{j: 0 \leq j \leq t-1, e_{a+j b}=+1\right\}\right| \\
& -\left|\left\{j: 0 \leq j \leq t-1, e_{j}=-1\right\}\right| \\
= & (t-h)-h=t-2 h .
\end{aligned}
$$

(2.17) holds with probability $\frac{1}{2^{t}}\left(\begin{array}{l}t \\ h\end{array}\right)$ so that

$$
P\left(\left|\sum_{j=0}^{t-1} e_{a+j b}\right|>M\right)=\sum_{h:|t-2 h|>M} \frac{1}{2^{t}}\left(\begin{array}{l}
t \\
h
\end{array}\right)=\frac{1}{2^{t}} \sum_{h:|h-t / 2|>M / 2}\left(\begin{array}{l}
t \\
h
\end{array}\right)
$$

An easy computation shows that if $t \rightarrow \infty$ and $k \leq t^{2 / 3}$, then

$$
\left(\begin{array}{c}
t \\
{[t / 2]-k}
\end{array}\right)=\left(\begin{array}{c}
t \\
{[t / 2]}
\end{array}\right) \exp \left(-\frac{2 k^{2}}{t}+O\left(\frac{k^{3}}{t^{2}}\right)\right) .
$$

If we also use the fact that $\left(\begin{array}{l}t \\ i\end{array}\right)$ is increasing in $i$ for $0 \leq i \leq t / 2$, it follows easily that for $N$ large enough (so that $t$ is also large by (2.15)), 


$$
\begin{aligned}
\sum_{h:|h-t / 2|>M / 2}\left(\begin{array}{l}
t \\
h
\end{array}\right) & =\sum_{h:|h-t / 2|>3(t \log t)^{1 / 2}}\left(\begin{array}{l}
t \\
h
\end{array}\right) \\
& <\left(\begin{array}{c}
t \\
{[t / 2]}
\end{array}\right) t \exp \left(-2\left(3(t \log t)^{1 / 2}\right)^{2} \frac{1}{t}+o(1)\right) \\
& =\left(\begin{array}{c}
t \\
{[t / 2]}
\end{array}\right) t \exp (-18 \log t+o(1))<\frac{2^{t}}{t^{16}}
\end{aligned}
$$

Since $M \leq L$, it follows from (2.15), (2.18) and (2.19) that

$$
\begin{aligned}
P\left(\left|\sum_{j=0}^{t-1} e_{a+j b}\right|>L\right) & \leq P\left(\left|\sum_{j=0}^{t-1} e_{a+j b}\right|>M\right) \\
& <\frac{1}{2^{t}} \cdot \frac{2^{t}}{t^{16}}=\frac{1}{t^{16}}<\frac{1}{L^{16}}=o\left(\frac{1}{N^{8}}\right)<\frac{\varepsilon}{N^{3}},
\end{aligned}
$$

which proves (2.14) and this completes the proof of (2.2).

Proof of Theorem 2. First we prove (2.3). Since by (1.2), for $N>2 k$,

$$
\begin{aligned}
C_{k}\left(E_{N}\right) & \geq\left|V\left(E_{N},[N / 2]-k,(0,1, \ldots, k-2,[N / 2])\right)\right| \\
& =\left|\sum_{n=1}^{[N / 2]-k} e_{n} e_{n+1} \ldots e_{n+k-2} e_{n+[N / 2]}\right|
\end{aligned}
$$

we have

$$
P\left(C_{k}\left(E_{N}\right)>\delta N^{1 / 2}\right) \geq P\left(\left|\sum_{n=1}^{[N / 2]-k} e_{n} e_{n+1} \ldots e_{n+k-2} e_{n+[N / 2]}\right|>\delta N^{1 / 2}\right)
$$

so that it suffices to prove

$$
P\left(\left|\sum_{n=1}^{[N / 2]-k} e_{n} e_{n+1} \ldots e_{n+k-2} e_{n+[N / 2]}\right|>\delta N^{1 / 2}\right)>1-\varepsilon .
$$

For any fixed $(k-1)$-tuple $\mathbf{u}=\left(e_{n}, e_{n+1}, \ldots, e_{n+k-2}\right)$, write $f_{n}=e_{n} e_{n+1} \ldots$ $\ldots e_{n+k-2}$, and define $g_{n}$ by

$$
e_{n+[N / 2]}=f_{n} g_{n}
$$

(so that $g_{n} \in\{-1,+1\}$ ). Then the sum in $(2.20)$ can be rewritten as

$$
\sum_{n=1}^{[N / 2]-k} e_{n} e_{n+1} \ldots e_{n+k-2} e_{n+[N / 2]}=\sum_{n=1}^{[N / 2]-k} g_{n}
$$

Since $e_{n+[N / 2]}$ assumes the values -1 and +1 , independently of $e_{1}, e_{2}, \ldots$ $\ldots, e_{[N / 2]}$, with probability $1 / 2$, so clearly does $g_{n}$. Thus $(2.20)$ can be writ- 
ten in the equivalent form

$$
P\left(\left|\sum_{n=1}^{[N / 2]-k} g_{n}\right|>\delta N^{1 / 2}\right)>1-\varepsilon
$$

where $g_{1}, \ldots, g_{[N / 2]-k}$ are independent and assume the values -1 and +1 with probability $1 / 2$. Writing again

$$
\left|\left\{n: 1 \leq n \leq[N / 2]-k, e_{n}=-1\right\}\right|=h,
$$

we deduce in the same way as in the proof of (2.1) that

$$
\sum_{n=1}^{[N / 2]-k} g_{n}=[N / 2]-k-2 h
$$

and

$$
\begin{aligned}
P\left(\left|\sum_{n=1}^{[N / 2]-k} g_{n}\right|>\delta N^{1 / 2}\right) & =\sum_{h:|[N / 2]-k-2 h|>\delta N^{1 / 2}} \frac{1}{2^{[N / 2]-k}}\left(\begin{array}{c}
{[N / 2]-k} \\
h
\end{array}\right) \\
= & \sum_{h:|(1 / 2)([N / 2]-k)-h|>(\delta / 2) N^{1 / 2}} \frac{1}{2^{[N / 2]-k}}\left(\begin{array}{c}
{[N / 2]-k} \\
h
\end{array}\right) .
\end{aligned}
$$

For fixed $k$, small enough $\delta=\delta(\varepsilon)$ and $N>N_{0}(\varepsilon, k)$, this is, indeed, $>1-\varepsilon$. Since this lower bound is uniform for any choice of $e_{1}, e_{2}, \ldots, e_{[N / 2]},(2.20)$ also holds and this completes the proof of (2.3).

Now we prove (2.4). This will be an easy consequence of an upper bound for the sum

$$
S_{N, k}(l)=\sum_{E_{N} \in\{-1,+1\}^{N}} \sum_{M} \sum_{D}\left(V\left(E_{N}, M, D\right)\right)^{2 l}
$$

where the inner sums are taken over all $M \in \mathbb{N}, D=\left(d_{1}, \ldots, d_{k}\right)$ with $0 \leq d_{1}<\ldots<d_{k}, M+d_{k} \leq N$, and $l$ will be fixed later in terms of $k$ and $N$. The sum above can be rewritten as

$$
S_{N, k}(l)=\sum_{M} \sum_{D} Z(M, D)
$$

where

$$
\begin{aligned}
Z(M, D) & =\sum_{E_{n} \in\{-1,+1\}^{N}}\left(V\left(E_{N}, M, D\right)\right)^{2 l} \\
& =\sum_{E_{n} \in\{-1,+1\}^{N}}\left(\sum_{n=1}^{M} e_{n+d_{1}} e_{n+d_{2}} \ldots e_{n+d_{k}}\right)^{2 l} .
\end{aligned}
$$


If $M \leq N^{1 / 4}$ then clearly

$$
\begin{aligned}
Z(M, D) & =\sum_{E_{n} \in\{-1,+1\}^{N}}\left(V\left(E_{N}, M, D\right)\right)^{2 l} \\
& \leq \sum_{E_{n} \in\{-1,+1\}^{N}} M^{2 l}=2^{N} M^{2 l} .
\end{aligned}
$$

Assume now that

$$
N^{1 / 4}<M \leq N
$$

Write $e_{n+d_{1}} e_{n+d_{2}} \ldots e_{n+d_{k}}=x_{n}$. Then by the multinomial theorem we have

$$
Z(M, D)=\sum_{E_{N} \in\{-1,+1\}^{N}} \sum_{t=1}^{2 l} \sum_{\substack{1 \leq i_{1}<\ldots<i_{t} \leq M \\ j_{1}+\ldots+j_{t}=2 l \\ 1 \leq j_{1}, \ldots, j_{t}}} \frac{(2 l) !}{j_{1} ! \ldots j_{t} !} x_{i_{1}}^{j_{1}} \ldots x_{i_{t}}^{j_{t}} .
$$

Observe that each $x_{i} \in\{-1,+1\}$, and thus the value of $x_{i}^{j}$ depends only on the parity of $j: x_{i}^{j}=1$ if $j$ is even and $x_{i}^{j}=x_{i}$ if $j$ is odd. Let $Z_{1}$ denote the contribution of those terms for which at least one of $j_{1}, \ldots, j_{t}$ is odd and let $Z_{2}$ denote the contribution of the terms such that each of $j_{1}, \ldots, j_{t}$ is even, so that

$$
Z(M, D)=Z_{1}+Z_{2}
$$

All the terms in $Z_{1}$ can be replaced by a term of the form a constant times $x_{s_{1}} \ldots x_{s_{u}}$ where $u \leq 2 l, 1 \leq s_{1}<\ldots<s_{u} \leq M$. Thus $Z_{1}$ can be rewritten in the form

$$
Z_{1}=\sum_{u \leq 2 l} \sum_{1 \leq s_{1}<\ldots<s_{u} \leq M} a\left(s_{1}, \ldots, s_{u}\right) \sum_{E_{N} \in\{-1,+1\}^{N}} x_{s_{1}} \ldots x_{s_{u}}
$$

(where the coefficients $a\left(s_{1}, \ldots, s_{u}\right)$ are non-negative integers independent of $E_{N}$ ). Replace $x_{s_{i}}$ again by $e_{s_{i}+d_{1}} e_{s_{i}+d_{2}} \ldots e_{s_{i}+d_{k}}$ for each of $i=1, \ldots, u$; then each term $x_{s_{1}} \ldots x_{s_{u}}$ becomes of the form

$$
x_{s_{1}} \ldots x_{s_{u}}=e_{s_{1}+d_{1}} e_{v_{2}}^{q_{2}} e_{v_{3}}^{q_{3}} \ldots e_{v_{z}}^{q_{z}}
$$

where $s_{1}+d_{1}<v_{2}<\ldots<v_{z}$ and $q_{i} \in \mathbb{N}$ for $i=2,3, \ldots, z$. Then the innermost sum in $(2.25)$ is

$$
\sum_{\left(e_{1}, \ldots, e_{s_{1}+d_{1}-1}, e_{s_{1}+d_{1}+1}, \ldots, e_{N}\right) \in\{-1,+1\}^{N-1}} e_{v_{2}}^{q_{2}} \ldots e_{v_{z}}^{q_{z}} \sum_{e_{s_{1}+d_{1}} \in\{-1,+1\}} e_{s_{1}+d_{1}} .
$$

Here the inner sum is 0 so that the innermost sum in (2.25) is always 0 and thus

$$
Z_{1}=0 \text {. }
$$

In $Z_{2}$ we may replace each $j_{i}$ by $2 r_{i}$, and then we may use the fact that the inner sums are independent of $E_{N}$ : 


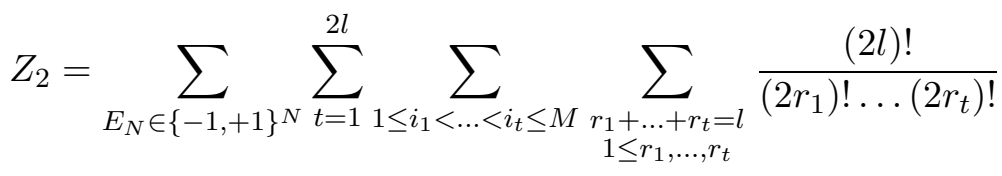

$$
\begin{aligned}
& =2^{N} \sum_{t=1}^{2 l} \sum_{1 \leq i_{1}<\ldots<i_{t} \leq M} \sum_{\substack{r_{1}+\ldots+r_{t}=l \\
1 \leq r_{1}, \ldots, r_{t}}} \frac{(2 l) !}{\left(2 r_{1}\right) ! \ldots\left(2 r_{t}\right) !} .
\end{aligned}
$$

To compute this sum observe that, by a similar argument,

$$
\begin{aligned}
F\left(y_{1}, \ldots, y_{M}\right) & :=\sum_{\left\{f_{1}, \ldots, f_{M}\right\} \in\{-1,+1\}^{M}}\left(f_{1} y_{1}+\ldots+f_{M} y_{M}\right)^{2 l} \\
& =2^{M} \sum_{t=1}^{2 l} \sum_{\substack { 1 \leq i_{1}<\ldots<i_{t} \leq M \\
\begin{subarray}{c}{r_{1}+\ldots+r_{t}=l \\
1 \leq r_{1}, \ldots, r_{t}{ 1 \leq i _ { 1 } < \ldots < i _ { t } \leq M \\
\begin{subarray} { c } { r _ { 1 } + \ldots + r _ { t } = l \\
1 \leq r _ { 1 } , \ldots , r _ { t } } }\end{subarray}} \frac{(2 l) !}{\left(2 r_{1}\right) ! \ldots\left(2 r_{t}\right) !} y_{i_{1}}^{2 r_{1}} \ldots y_{i_{t}}^{2 r_{t}} .
\end{aligned}
$$

Substituting $y_{1}=\ldots=y_{M}=1$, we obtain $F(1, \ldots, 1)=2^{M-N} Z_{2}$. On the other hand, $F(1, \ldots, 1)$ is easy to compute: if

$$
\left|\left\{f_{i}: 1 \leq i \leq M, f_{i}=-1\right\}\right|=h \text {, }
$$

then

$$
f_{1}+\ldots+f_{M}=M-2 h
$$

and there are $\left(\begin{array}{c}M \\ h\end{array}\right) M$-tuples satisfying (2.27). Thus

$$
2^{M-N} Z_{2}=F(1, \ldots, 1)=\sum_{h=0}^{M}\left(\begin{array}{c}
M \\
h
\end{array}\right)(M-2 h)^{2 l}=2 \sum_{h=0}^{[M / 2]}\left(\begin{array}{c}
M \\
h
\end{array}\right)(M-2 h)^{2 l} \text {. }
$$

Now we fix the value of $l$ : let

$$
l=[2 k \log N]
$$

Write

$$
A_{h}=\left(\begin{array}{c}
M \\
h
\end{array}\right)(M-2 h)^{2 l} \quad \text { so that } \quad 2^{M-N} Z_{2}=2 \sum_{h=0}^{[M / 2]} A_{h} .
$$

A little computation shows that for $h<M / 2$ we have

$$
\frac{A_{h+1}}{A_{h}}=\frac{M-h}{h+1}\left(1-\frac{2}{M-2 h}\right)^{2 l}
$$

and clearly this is decreasing on the interval $0<h \leq M / 2-1$. Thus writing $H=M / 2-\sqrt{l M}$, by (2.23) and (2.28), for $h \leq H$ we have

$$
\begin{aligned}
\frac{A_{h+1}}{A_{h}} & \geq \frac{M-H}{H+1}\left(1-\frac{2}{M-2 H}\right)^{2 l} \\
& =\frac{M / 2+\sqrt{l M}}{M / 2-\sqrt{l M}+1}\left(1-\frac{1}{\sqrt{l M}}\right)^{2 l}
\end{aligned}
$$




$$
\begin{aligned}
& =(1+(1+o(1)) 4 \sqrt{l / M})(1-(1+o(1)) 2 \sqrt{l / M}) \\
& =(1+(1+o(1)) 2 \sqrt{l / M})>1 .
\end{aligned}
$$

It follows that writing $H_{0}=[M / 2-\sqrt{l M}+1]$, we have $A_{0}<A_{1}<\ldots<A_{H_{0}}$, whence

$$
\begin{aligned}
2^{M-N} Z_{2} & =2 \sum_{h=0}^{[M / 2]} A_{h}=2\left(\sum_{h=0}^{H_{0}} A_{h}+\sum_{h=H_{0}+1}^{[M / 2]} A_{h}\right) \\
& <2\left(\sum_{h=0}^{H_{0}} A_{H_{0}}+\sum_{h=H_{0}+1}^{[M / 2]}\left(\begin{array}{c}
M \\
h
\end{array}\right)(M-2 h)^{2 l}\right) \\
& <2\left(2 H_{0} A_{H_{0}}+\left(M-2 H_{0}\right)^{2 l} \sum_{h=0}^{M}\left(\begin{array}{c}
M \\
h
\end{array}\right)\right) \\
& <2\left(M\left(\begin{array}{c}
M \\
H_{0}
\end{array}\right)\left(M-2 H_{0}\right)^{2 l}+\left(M-2 H_{0}\right)^{2 l} 2^{M}\right) \\
& <2^{M+1}(M+1)\left(M-2\left(\frac{M}{2}-\sqrt{l M}\right)\right)^{2 l} \\
& <2^{M+2} M(4 l M)^{l} \quad \text { for } N^{1 / 4}<M \leq N .
\end{aligned}
$$

It follows from $(2.21),(2.22),(2.24),(2.26)$ and $(2.29)$ that

$$
\begin{aligned}
(2.30) S_{N, k}(l) & =\sum_{D}\left(\sum_{M \leq N^{1 / 4}} Z(M, D)+\sum_{N^{1 / 4}<M \leq N} Z(M, D)\right) \\
& <\sum_{D}\left(\sum_{M \leq N^{1 / 4}} 2^{N} M^{2 l}+\sum_{N^{1 / 4}<M \leq N} 2^{N+2}(4 l)^{l} N^{l+1}\right) \\
& <\sum_{D}\left(\sum_{M \leq N^{1 / 4}} 2^{N} N^{l / 2}+N^{l+2} 2^{N+2}(4 l)^{l}\right) \\
& <2^{N} \sum_{D}\left(N^{l / 2+1 / 4}+4 N^{l+2}(4 l)^{l}\right) \\
& <5 \cdot 2^{N} N^{l+2}(4 l)^{l} \sum_{D} 1 .
\end{aligned}
$$

Each $d_{i}$ in $D=\left(d_{1}, \ldots, d_{k}\right)$ satisfies $d_{i} \in\{0,1, \ldots, N-1\}$ thus it can be chosen in at most $N$ ways so that

$$
\sum_{D} 1 \leq N^{k}
$$

It follows from (2.30) and (2.31) that

$$
S_{N, k}(l)<5 \cdot 2^{N} N^{k+l+2}(4 l)^{l} .
$$


On the other hand, writing $X=5(k N \log N)^{1 / 2}$, we clearly have

$$
\begin{aligned}
S_{N, k}(l) & =\sum_{E_{N} \in\{-1,+1\}^{N}} \sum_{M} \sum_{D}\left(V\left(E_{N}, M, D\right)\right)^{2 l} \\
& \geq \sum_{E_{N} \in\{-1,+1\}^{N}}\left(\max _{M, D}\left|V\left(E_{N}, M, D\right)\right|\right)^{2 l} \\
& =\sum_{E_{N} \in\{-1,+1\}^{N}}\left(C_{k}\left(E_{N}\right)\right)^{2 l} \\
& \geq X^{2 l}\left|\left\{E_{N}: E_{N} \in\{-1,+1\}^{N}, C_{k}\left(E_{N}\right)>X\right\}\right| .
\end{aligned}
$$

It follows from (2.28), (2.32) and (2.33) that

$$
\begin{aligned}
P\left(C_{k}\left(E_{N}\right)>X\right) & =\frac{1}{2^{N}}\left|\left\{E_{N}: E_{N} \in\{-1,+1\}^{N}, C_{k}(N)>X\right\}\right| \\
& \leq 5 N^{k+l+2}(4 l)^{l} X^{-2 l} \\
& =5 N^{k+l+2}(4 l)^{l}(25 k N \log N)^{-l}<5 N^{k+2} 3^{-l} \\
& =15 N^{k+2} 3^{-l-1}<15 N^{2 k} 3^{-2 k} \log N \\
& =15 N^{2 k(1-\log 3)}<15 N^{1-\log 3}
\end{aligned}
$$

and this is $<\varepsilon$ if $N$ is large enough in terms of $\varepsilon$ (since $1-\log 3<0$ ), which completes the proof of (2.4).

\section{The minimum of the PR measures. Write}

$$
m(N)=\min _{E_{N} \in\{-1,+1\}^{N}} W\left(E_{N}\right), \quad M_{k}(N)=\min _{E_{N} \in\{-1,+1\}^{N}} C_{k}\left(E_{N}\right) .
$$

The estimate of $m(N)$ is a classical problem. In 1964 Roth [Ro] proved that $m(N)>c_{1} N^{1 / 4}$ for some positive absolute constant $c_{1}$. From the opposite side Erdős, Spencer, Sárközy and Beck estimated $m(N)$, and finally in 1996 Matoušek and Spencer [MSp] showed that $m(N)<c_{2} N^{1 / 4}$ so that now the order of magnitude of $m(N)$ is known.

On the other hand, as far as we know $M_{k}(N)$ has not been studied yet, not even $M_{2}(N)$ has been estimated. We will prove

Theorem 3. (i) For $k, N \in \mathbb{N}, 2 \leq k \leq N$ we have

$$
M_{k}(N)<27 k N^{1 / 2} \log N \text {. }
$$

(ii) For $k \in \mathbb{N}, k \geq 2$ there is a number $N_{0}(k)$ such that if $N \in \mathbb{N}$, $N>N_{0}$, then also

$$
M_{k}(N) \leq 5(k N \log N)^{1 / 2} .
$$

On the other hand, we have only a very weak lower bound for $M_{k}(N)$ and only in the case when $k$ is even: 
Theorem 4. If $k, N \in \mathbb{N}, k$ is even, $2 \leq k \leq N$, then

$$
M_{k}(N) \geq\left[\frac{1}{\log 2}(\log N-\log k)\right] .
$$

Note that if $k$ is odd then there is no lower bound of type (3.3). More exactly we have $M_{k}(N)=1$ for all $N \in \mathbb{N}$ and odd $k$ with $1<k \leq N$. Indeed, $M_{k}(N) \geq 1$ is trivial. To see that also $M_{k}(N) \leq 1$, consider the sequence $E_{N}=\left\{e_{1}, \ldots, e_{N}\right\} \in\{-1,+1\}^{N}$ defined by $e_{n}=(-1)^{n}$ for $n=1, \ldots, N$. Then for all $n$ and $D=\left(d_{1}, \ldots, d_{k}\right)$ we have

$$
\begin{aligned}
e_{n+1+d_{1}} \ldots e_{n+1+d_{k}} & =\left(-e_{n+d_{1}}\right) \ldots\left(-e_{n+d_{k}}\right) \\
& =(-1)^{k} e_{n+d_{1}} \ldots e_{n+d_{k}}=-e_{n+d_{1}} \ldots e_{n+d_{k}}
\end{aligned}
$$

whence, for all $M, D$,

$$
\left|V\left(E_{N}, M, D\right)\right|=\left|\sum_{n=1}^{M} e_{n+d_{1}} \ldots e_{n+d_{k}}\right|= \begin{cases}0 & \text { if } M \text { is even, } \\ 1 & \text { if } M \text { is odd }\end{cases}
$$

so that

$$
C_{k}\left(E_{N}\right)=\max _{M, D}\left|V\left(E_{N}, M, D\right)\right|=1 \quad \text { for } k \text { odd, }
$$

which proves $M_{k}(N) \leq 1$.

We remark that it is easy to see that in this example if $k$ is even, then $C_{k}\left(E_{N}\right)$ is large:

$$
\begin{aligned}
C_{k}\left(E_{N}\right) & =\left|V\left(E_{N}, N-k+1,(0,1, \ldots, k-1)\right)\right| \\
& =\left|\sum_{n=1}^{N-k+1}(-1)^{n+(n+1)+\ldots+(n-k+1)}\right| \\
& =| \pm(N-k+1)|=N-k+1 \quad \text { for } k \text { even. }
\end{aligned}
$$

The contrast between the sizes of $C_{2}\left(E_{N}\right)$ and $C_{3}\left(E_{N}\right)$ in the example above inspires the following problem that we have not been able to settle:

Problem 1. For $N \rightarrow \infty$, are there sequences $E_{N}$ such that $C_{2}\left(E_{N}\right)=$ $O(\sqrt{N})$ and $C_{3}\left(E_{N}\right)=O(1)$ simultaneously?

We think that the upper bounds in Theorem 3 are much closer to the truth than the lower bound in Theorem 4 but, unfortunately, we have not been able to tighten the gap. In particular, we have not been able to settle the following problem:

Problem 2. Is it true that there is a $c>0$ such that as $N \rightarrow \infty$,

$$
M_{2}(N) \gg N^{c} ?
$$

We think that the answer is affirmative. We will return to this problem at the end of the proof of Theorem 4 . 
Proof of Theorem 3. (i) Let $p$ denote the smallest prime with $p>N$ so that, by Chebyshev's theorem,

$$
N<p \leq 2 N .
$$

Define $E_{N}=\left\{e_{1}, \ldots, e_{N}\right\} \in\{-1,+1\}^{N}$ by

$$
e_{n}=\left(\frac{n}{p}\right) \quad \text { for } n=1, \ldots, N
$$

where $\left(\frac{n}{p}\right)$ denotes the Legendre symbol. By Theorem 1, formula (3.1) in Part I [MSá] for this sequence $E_{N}$ we have

$$
C_{k}\left(E_{N}\right) \leq 9 k p^{1 / 2} \log p
$$

whence, by (3.5),

$$
C_{k}\left(E_{N}\right) \leq 9 k(2 N)^{1 / 2} \log (2 N)<27 k N^{1 / 2} \log N
$$

which proves (3.1).

(ii) It follows from (2.4) in Theorem 2 (with, say, $\varepsilon=1 / 2$ ) that for $N>N_{0}(k)$ there is at least one $E_{N} \in\{-1,+1\}^{N}$ with

$$
C_{k}\left(E_{N}\right) \leq 5(k N \log N)^{1 / 2},
$$

which proves (3.2).

Proof of Theorem 4. First we remark that (3.3) is always true for $k \geq$ $N / 2$ so that we can now suppose

$$
k \leq N / 2 .
$$

Write

$$
Q=\left[\frac{1}{\log 2}(\log N-\log k)\right]
$$

so that $Q \geq 1$ by (3.6). Let $E_{N}=\left\{e_{1}, \ldots, e_{N}\right\} \in\{-1,+1\}^{N}$ and consider the $N-Q+1 Q$-tuples

$$
\mathbf{v}_{1}=\left(e_{1}, \ldots, e_{Q}\right), \mathbf{v}_{2}=\left(e_{2}, \ldots, e_{Q+1}\right), \ldots, \mathbf{v}_{N-Q+1}=\left(e_{N-Q+1}, \ldots, e_{N}\right) .
$$

We will show that there are subscripts

$$
(1 \leq) i_{1}<\ldots<i_{k}(\leq N-Q+1)
$$

with

$$
\mathbf{v}_{i_{1}}=\ldots=\mathbf{v}_{i_{k}} .
$$

The number of distinct $Q$-tuples in $\{-1,+1\}^{Q}$ is $2^{Q}$. Thus if the number of the vectors $\mathbf{v}_{i}$ is greater than $(k-1) 2^{Q}$, then by the pigeon-hole principle there is at least one $Q$-tuple occurring at least $k$ times, so that it suffices to show that

$$
N-Q+1>(k-1) 2^{Q} .
$$


By $Q \geq 1$ and the definition of $Q$ we have

$$
\begin{aligned}
(k-1) 2^{Q}+Q-1 & <(k-1) 2^{Q}+2^{Q}-1<k \cdot 2^{Q} \\
& =k \exp (Q \log 2) \leq k \exp (\log N-\log k)=N,
\end{aligned}
$$

whence (3.9) follows so that indeed there are $i_{1}, \ldots, i_{k}$ satisfying (3.7) and (3.8).

Now write $d_{j}=i_{j}-1$ for $j=1, \ldots, k$ and $D=\left(d_{1}, \ldots, d_{k}\right)$, and consider the sum

$$
V\left(E_{N}, Q, D\right)=\sum_{n=1}^{Q} e_{n+d_{1}} e_{n+d_{2}} \ldots e_{n+d_{k}} .
$$

By (3.8) and the definition of the vectors $\mathbf{v}_{1}, \ldots, \mathbf{v}_{N-Q+1}$, for $n=1, \ldots, Q$ we have

$$
e_{n+d_{1}} e_{n+d_{2}} \ldots e_{n+d_{k}}=e_{i_{1}+(n-1)} e_{i_{2}+(n-1)} \ldots e_{i_{k}+(n-1)}=\left(e_{i_{1}+(n-1)}\right)^{k} .
$$

But $e_{i_{1}+(n-1)} \in\{-1,+1\}$ and $k$ is even and thus this equals 1 . It follows that

$$
V\left(E_{N}, Q, D\right)=\sum_{n=1}^{Q} 1=Q
$$

so that, by (1.2),

$$
C_{k}\left(E_{N}\right) \geq\left|V\left(E_{N}, Q, D\right)\right|=Q
$$

for all $E_{N} \in\{-1,+1\}^{N}$, which proves (3.3).

We have made a considerable effort to settle Problem 2 above. Even related computer calculations have been carried out. We computed $M_{2}(83)=$ 10 (see also Table 1 ). However, to gather computer evidence of any value one has to consider much greater values of $N$, which again seems very difficult.

Of course, it is possible that a simple argument has been overlooked by us and by those who also approached the problem (we asked several people). However, there are certain signs which seem to indicate that, perhaps, the problem is really difficult. E.g., in the case $k=2$ one might like to improve on the argument used in the proof of Theorem 4 in the following way: again consider the $Q$-tuples $\mathbf{v}_{1}, \ldots, \mathbf{v}_{N-Q+1}$ defined there but now take $Q$ much greater, say, $Q \sim N^{c^{\prime}}$ for some $c^{\prime}>0$. A similar argument shows that it would suffice to find $i<j$ such that the scalar product of the vectors $\mathbf{v}_{i}, \mathbf{v}_{j}$ is "large". This is the same as finding two of the given vectors so that the angle between them is "significantly less" than $\pi / 2$. However, this approach fails since "many" vectors can be given with the property that the angle between any two of them is "large"; see $[\mathrm{EF}]$ for further details. 
Table 1

\begin{tabular}{cc}
\hline$N$ & $M_{2}(N)$ \\
\hline $2 \leq N \leq 3$ & 1 \\
$4 \leq N \leq 6$ & 2 \\
$7 \leq N \leq 11$ & 3 \\
$12 \leq N \leq 17$ & 4 \\
$18 \leq N \leq 26$ & 5 \\
$27 \leq N \leq 39$ & 6 \\
$40 \leq N \leq 44$ & 7 \\
$45 \leq N \leq 55$ & 8 \\
$56 \leq N \leq 68$ & 9 \\
$69 \leq N \leq 83$ & 10 \\
$84 \leq N \leq 93$ & 10 or 11 \\
$94 \leq N \leq 106$ & between 10 and 12 \\
$107 \leq N \leq 121$ & between 10 and 13 \\
$122 \leq N \leq 134$ & between 10 and 14 \\
\hline
\end{tabular}

Another warning sign is that Problem 1 is related to the classical and very difficult problem on the maximal absolute value of polynomials with -1 and +1 coefficients on the unit circle (see, e.g., [Kah, pp. 75-78]). Indeed, the study of the fourth mean of such a polynomial leads to sums of the type occurring in the definition of $C_{2}\left(E_{N}\right)$.

Our results above inspire a further problem:

Problem 3. What is the connection between $M_{2}(N)$ and $M_{4}(N)$ ? Is it true that for $N>N_{0}$ we have $M_{4}(N)>M_{2}(N)$ ? Perhaps, even $M_{4}(N)-$ $M_{2}(N) \rightarrow \infty$ as $N \rightarrow \infty$.

4. Comparison of correlations of different orders. First we will show that if $k \in \mathbb{N}, l \in \mathbb{N}, k \mid l, N \rightarrow \infty$ and $C_{l}\left(E_{N}\right)$ is "small", more exactly, $C_{l}\left(E_{N}\right)=o(N)$, then $C_{k}\left(E_{N}\right)$ is also small:

Theorem 5. For $k, l, N \in \mathbb{N}, k \mid l, E_{N} \in\{-1,+1\}^{N}$ we have

$$
C_{k}\left(E_{N}\right) \leq N\left(\frac{(l !)^{k / l}}{k !}\left(\frac{C_{l}\left(E_{N}\right)}{N}\right)^{k / l}+\left(\frac{l^{2}}{N}\right)^{k / l}\right) .
$$

Next we will show that in the assertion of the first paragraph of this section the condition $k \mid l$ is necessary and, indeed, for any fixed $k$ and for $N \rightarrow \infty$ there is an $E_{N} \in\{-1,+1\}^{N}$ such that $C_{l}\left(E_{N}\right)$ is small when $k \nmid l$, whereas $C_{k}\left(E_{N}\right)$ is large $(\gg N)$ :

Theorem 6. If $k, N \in \mathbb{N}$, and $k \leq N$, then there is a sequence $E_{N} \in$ $\{-1,+1\}^{N}$ such that if $l \in \mathbb{N}, l \leq N / 2$, then

$$
\begin{aligned}
& C_{l}\left(E_{N}\right)>(N-l) / k-54 k^{2} N^{1 / 2} \log N \quad \text { if } k \mid l, \\
& C_{l}\left(E_{N}\right)<27 k^{2} l N^{1 / 2} \log N \quad \text { if } k \nmid l \text {. }
\end{aligned}
$$


Proof of Theorem 5. By (1.2), it suffices to prove that for all $M$ and $D=\left(d_{1}, \ldots, d_{k}\right)\left(\right.$ with $\left.0 \leq d_{1}<\ldots<d_{k}, M+d_{k} \leq N\right)$ we have

$$
\left|V\left(E_{N}, M, D\right)\right| \leq\left(\frac{(l !)^{k / l}}{k !}\left(\frac{C_{l}\left(E_{N}\right)}{N}\right)^{k / l}+\left(\frac{l^{2}}{N}\right)^{k / l}\right) .
$$

Write $l / k=t$ so that $t \in \mathbb{N}$ because $k \mid l$. Then clearly,

$$
\begin{aligned}
V\left(E_{N}, M, D\right)^{t} & =\left(\sum_{n_{1}=1}^{M} e_{n_{1}+d_{1}} \ldots e_{n_{1}+d_{k}}\right) \ldots\left(\sum_{n_{t}=1}^{M} e_{n_{t}+d_{1}} \ldots e_{n_{t}+d_{k}}\right) \\
& =\sum_{n_{1}=1}^{M} \ldots \sum_{n_{t}=1}^{M} e_{n_{1}+d_{1}} \ldots e_{n_{1}+d_{k}} \ldots e_{n_{t}+d_{1}} \ldots e_{n_{t}+d_{k}} \\
& =S_{1}+S_{2}
\end{aligned}
$$

where $S_{1}$ denotes the contribution of those terms $e_{n_{1}+d_{1}} \ldots e_{n_{t}+d_{k}}$ where there are two equal subscripts:

$$
n_{i}+d_{u}=n_{j}+d_{v},
$$

while in $S_{2}$ all the subscripts are distinct.

First we estimate $S_{1}$. In (4.5), $u$ and $v$ can be chosen in at most $k$ ways, $i, j$ in $t$ ways, $n_{j}$ (for fixed $j$ ) in $M$ ways, and $u, v, n_{j}$ determine $n_{i}$ uniquely. Each of the $t-2$ remaining $n_{h}$ 's can be chosen in at most $M$ ways, so that $S_{1}$ has at most $k^{2} t^{2} M \cdot M^{t-2}=l^{2} M^{t-1}$ terms and thus

$$
\left|S_{1}\right| \leq l^{2} M^{t-1} \text {. }
$$

Now we estimate $S_{2}$. Consider each of the terms $e_{n_{1}+d_{1}} \ldots e_{n_{t}+d_{k}}$ in $S_{2}$, and rearrange the order of the factors $e_{n_{i}+d_{u}}$ so that the subscripts should be increasing:

$$
e_{n_{1}+d_{1}} \ldots e_{n_{t}+d_{k}}=e_{i_{1}} \ldots e_{i_{l}}, \quad i_{1}<\ldots<i_{l} .
$$

Now we $t$-colour these factors $e_{i_{1}}, \ldots, e_{i_{l}}$ : if the subscript of $e_{i_{u}}$ is of the form $i_{u}=n_{j}+d_{v}$, then we colour $e_{i_{u}}$ by the $j$ th colour. Then to each term $e_{i_{1}} \ldots e_{i_{l}}$ we may assign the sequence of the colours following each other in the order used to colour $e_{i_{1}}, \ldots, e_{i_{l}}$. In this way we get colour patterns of length $l$ where each of the $t$ colours occurs $k$ times, so that the number of these colour patterns is $l ! /(k !)^{t}$.

Now fix any of the colour patterns, and consider each of the terms $e_{i_{1}} \ldots e_{i_{l}}$ with this fixed colour pattern. We define an equivalence relation among these terms: we say that

$$
e_{i_{1}} \ldots e_{i_{l}} \sim e_{j_{1}} \ldots e_{j_{l}} \quad \text { if } \quad j_{1}-i_{1}=\ldots=j_{l}-i_{l} .
$$

Clearly, this is indeed an equivalence relation. Now fix a colour pattern and an equivalence class, and collect all the terms from this class. Let $e_{h_{1}} \ldots e_{h_{l}}$ 
$\left(h_{1}<\ldots<h_{l}\right)$ be the term for which the first subscript is minimal; it is easy to see that $h_{1}=1+d_{1}$. Write

$$
h_{i}-1=f_{i} \quad \text { for } i=1, \ldots, l,
$$

and let $Q$ denote the number of terms in the given equivalence class. Then it is easy to see that the terms in this equivalence class are $e_{n+f_{1}} \ldots e_{n+f_{l}}$ with $n=1, \ldots, Q$ so that, by (1.2), the absolute value of the sum of the terms in this class is

$$
\left|\sum_{n=1}^{Q} e_{n+f_{1}} \ldots e_{n+f_{l}}\right|=\left|V\left(E_{N}, Q,\left(f_{1}, \ldots, f_{l}\right)\right)\right| \leq C_{l}\left(E_{N}\right) .
$$

It remains to estimate the number of equivalence classes. An equivalence class is uniquely determined by the colour pattern, which can be chosen in $l ! /(k !)^{t}$ ways, and by the subscripts of the $t e_{h_{i}}$ 's where these colours first appear. The first of these subscripts, $h_{1}=1+d_{1}$, is fixed, while each of the other $t-1$ subscripts can be chosen in at most $M$ ways. Thus the number of equivalence classes is $\leq\left(l ! /(k !)^{t}\right) M^{t-1}$, and thus the total sum is

$$
\left|S_{2}\right| \leq \frac{l !}{(k !)^{t}} M^{t-1} C_{l}\left(E_{N}\right) .
$$

It follows from (4.4), (4.6) and (4.7) that

$$
\begin{aligned}
& \left|V\left(E_{N}, M, D\right)\right| \leq\left|S_{1}+S_{2}\right|^{1 / t} \leq\left(\left|S_{1}\right|+\left|S_{2}\right|\right)^{1 / t} \\
& \quad \leq\left(l^{2} M^{t-1}+\frac{l !}{(k !)^{t}} M^{t-1} C_{l}\left(E_{N}\right)\right)^{1 / t} \leq\left(l^{2} N^{t-1}+\frac{l !}{(k !)^{t}} N^{t-1} C_{l}\left(E_{N}\right)\right)^{1 / t} \\
& \quad=N\left(\frac{l^{2}}{N}+\frac{l !}{(k !) t} \cdot \frac{C_{l}\left(E_{N}\right)}{N}\right)^{1 / t} \leq N\left(\left(\frac{l^{2}}{N}\right)^{1 / t}+\frac{(l !)^{1 / t}}{k !}\left(\frac{C_{l}\left(E_{N}\right)}{N}\right)^{1 / t}\right)
\end{aligned}
$$

which proves (4.3) and this completes the proof of Theorem 5 .

Proof of Theorem 6. We will construct a sequence $E_{N} \in\{-1,+1\}^{N}$ with the desired properties. The construction will be based on the following result which was the crucial tool also in [MSá]:

Lemma 1. Suppose $p$ is a prime number, $F_{p}$ denotes the field of residue classes modulo $p, \bar{F}_{p}$ denotes the algebraic closure of $F_{p}, f(x) \in F_{p}[x]$ is a polynomial of degree $d$ which is not of the form $f(x)=b(g(x))^{2}$ with $b \in F_{p}, g(x) \in F_{p}[x]$ (in other words, if we factorize $f$ in $\bar{F}_{p}: f(x)=$ $b\left(x-x_{1}\right)^{d_{1}} \ldots\left(x-x_{s}\right)^{d_{s}}$, where $x_{i} \neq x_{j}$ for $i \neq j$, then there is at least one odd exponent $\left.d_{i}\right), X, Y$ are real numbers with $0<Y \leq p,\left(\frac{n}{p}\right)$ denotes the Legendre symbol for $p \nmid n$ and we write $\left(\frac{n}{p}\right)=0$ for $p \mid n$. Then

$$
\left|\sum_{X<n \leq X+Y}\left(\frac{f(n)}{p}\right)\right|<9 d p^{1 / 2} \log p .
$$


Proof. This is Corollary 1 in [MSá] and, indeed, we derived it from A. Weil's theorem [We].

Now let $p$ denote the smallest prime with $p>N$ so that, by Chebyshev's theorem,

$$
N<p \leq 2 N,
$$

and define $E_{N}=\left\{e_{1}, \ldots, e_{N}\right\} \in\{-1,+1\}^{N}$ by

$$
e_{n}= \begin{cases}\left(\frac{n}{p}\right) & \text { for } k \nmid n, \\ \left(\frac{(n-1)(n-2) \ldots(n-k+1)}{p}\right) & \text { for } k \mid n .\end{cases}
$$

First we prove (4.1). Assume that $k \mid l$. Then

$$
\begin{aligned}
C_{l}\left(E_{N}\right) & =\max _{M, D}\left|V\left(E_{N}, M, D\right)\right| \geq\left|V\left(E_{N}, N-l+1,(0,1, \ldots, l-1)\right)\right| \\
& =\left|\sum_{n=1}^{N-l+1} e_{n} e_{n+1} \ldots e_{n+l-1}\right|=\left|\sum_{r=1}^{k} S(r)\right|
\end{aligned}
$$

where $S(r)$ is defined by

$$
S(r)=\sum_{\substack{1 \leq n \leq N-l+1 \\ n \equiv r(\bmod k)}} e_{n} e_{n+1} \ldots e_{n+l+1} .
$$

Consider first the case $r=1$ :

$$
\begin{aligned}
S(1) & =\sum_{\substack{1 \leq n \leq N-l+1 \\
n \equiv 1(\bmod k)}} e_{n} e_{n+1} \ldots e_{n+l+1} \\
& =\sum_{\substack{1 \leq n \leq N-l+1 \\
n \equiv 1(\bmod k)}} \prod_{\substack{n+k-1 \leq m \leq n+l-1 \\
k \mid m}} e_{m-k+1} e_{m-k+2} \ldots e_{m} .
\end{aligned}
$$

For $k \mid m, 1 \leq m \leq N<p$ we have

$$
\begin{aligned}
& e_{m-k+1} e_{m-k+2} \ldots e_{m} \\
&=\left(\frac{m-k+1}{p}\right)\left(\frac{m-k+2}{p}\right) \ldots\left(\frac{m-1}{p}\right) \\
& \times\left(\frac{(m-1)(m-2) \ldots(m-k+1)}{p}\right) \\
&=\left(\frac{(m-1)^{2}(m-2)^{2} \ldots(m-k+1)^{2}}{p}\right)=+1 \quad \text { for } k \mid m
\end{aligned}
$$


so that

$$
S(1)=\sum_{\substack{1 \leq n \leq N-l+1 \\ n \equiv 1(\bmod k)}} 1=\left[\frac{N-l}{k}\right]+1>\frac{N-l}{k} .
$$

Consider now the case $2 \leq r \leq k$. Write each $n$ with $1 \leq n \leq N-l+1$, $n \equiv r(\bmod k)$ in the form $n=u k+r$ so that $S(r)$ can be rewritten as

$$
S(r)=\sum_{0 \leq u \leq(N-l+1-r) / k} e_{u k+r} e_{u k+r+1} \ldots e_{u k+r+l-1} .
$$

By (4.11), the product of the $e_{i}$ 's with $(u+1) k<i \leq(u+l / k) k$ in the term $e_{u k+r} e_{u k+r+1} \ldots e_{u k+r+l-1}$ is 1 so that these $e_{i}$ 's can be dropped. By using the definition of $e_{n}$ we get

$$
\begin{aligned}
S(r)= & \sum_{0 \leq u \leq(N-l+1-r) / k} e_{u k+r} \ldots e_{(u+1) k} e_{u k+l+1} \ldots e_{u k+r+l-1} \\
= & \sum_{0 \leq u \leq(N-l+1-r) / k}\left(\frac{u k+r}{p}\right) \ldots\left(\frac{((u+1) k-k+1) \ldots((u+1) k-1)}{p}\right) \\
& \times\left(\frac{u k+l+1}{p}\right) \ldots\left(\frac{u k+r+l-1}{p}\right) .
\end{aligned}
$$

Using the multiplicativity of the Legendre symbol, and then dropping the square factors in the "numerator" of the Legendre symbol, we can rewrite the last sum as

$S(r)=$

$\sum_{0 \leq u \leq(N-l+1-r) / k}\left(\frac{(u k+1) \ldots(u k+r-1)(u k+l+1) \ldots(u k+l+r-1)}{p}\right)$.

Since $k \leq N<p$, there is an integer $\bar{k}$ with

$$
k \bar{k} \equiv 1(\bmod p) \text {. }
$$

Then multiplying the sum above by $\left(\frac{\bar{k}^{2 r-2}}{p}\right)=1$ we get

$$
S(r)=\sum_{0 \leq u \leq(N-l+1-r) / k}\left(\frac{f(u)}{p}\right)
$$

where

$f(u)=(u+\bar{k})(u+2 \bar{k}) \ldots(u+(r-1) \bar{k})(u+(l+1) \bar{k}) \ldots(u+(l+r-1) \bar{k})$.

As $l+r-1<l+k \leq 2 l \leq N<p$, here all the zeros $-\bar{k},-2 \bar{k}, \ldots,-(l+r-1) \bar{k}$ are distinct modulo $p$, thus the polynomial $f$ satisfies the conditions in Lemma 1 so that the lemma can be applied to estimate this sum $S(r)$. 
The degree of $f(u)$ is $d=2(r-1)<2 k$ so that by Lemma 1 and (4.8),

$$
\begin{aligned}
|S(r)| & <9 \cdot 2 k p^{1 / 2} \log p \\
& \leq 18 k(2 N)^{1 / 2} \log 2 N<54 k N^{1 / 2} \log N \quad \text { for } 2 \leq r \leq k .
\end{aligned}
$$

It follows from (4.10), (4.12) and (4.14) that

$$
C_{l}\left(E_{N}\right) \geq S(1)-\sum_{r=2}^{k}|S(r)|>\frac{N-l}{k}-54 k^{2} N^{1 / 2} \log N,
$$

which completes the proof of (4.1).

(4.2) can be proved similarly. Assume that $k \nmid l$. By (1.2) it suffices to prove that for all $M \in \mathbb{N}, D=\left(d_{1}, \ldots, d_{l}\right)$ with $0 \leq d_{1}<\ldots<d_{l}, M+d_{l} \leq$ $N$ we have

$$
\left|V\left(E_{N}, M, D\right)\right|=\left|\sum_{n=1}^{M} e_{n+d_{1}} e_{n+d_{2}} \ldots e_{n+d_{l}}\right|<27 k^{2} l N^{1 / 2} \log N
$$

As in the proof of (4.1), we write

$$
V\left(E_{N}, M, D\right)=\sum_{r=1}^{k} S(r)
$$

where

$$
S(r)=\sum_{\substack{1 \leq n \leq M \\ n \equiv r(\bmod k)}} e_{n+d_{1}} e_{n+d_{2}} \ldots e_{n+d_{l}} .
$$

Again we substitute $n=u k+r$ so that

$$
S(r)=\sum_{0 \leq u \leq(M-r) / k} e_{u k+r+d_{1}} e_{u k+r+d_{2}} \ldots e_{u k+r+d_{l}}
$$

where, by (4.9),

$$
e_{u k+r+d_{i}}=\left(\frac{u k+r+d_{i}}{p}\right) \quad \text { for } k \nmid\left(r+d_{i}\right)
$$

and

$$
\begin{aligned}
& e_{u k+r+d_{i}} \\
& =\left(\frac{\left(u k+r+d_{i}-1\right)\left(u k+r+d_{i}-2\right) \ldots\left(u k+r+d_{i}-k+1\right)}{p}\right) \\
& \text { for } k \mid\left(r+d_{i}\right) .
\end{aligned}
$$

Using again the multiplicativity of the Legendre symbol, we can write the general term in the sum (4.17) as a single Legendre symbol whose "numerator" is a polynomial in $u$ which is the product of linear polynomials of the form $u k+a_{z}$ where, clearly, $1 \leq a_{z}<p$ so that for distinct values of $a_{z}$ these linear polynomials are also distinct modulo $p$. Clearly, each of these linear 
polynomials occurs at most twice, and $u k+a_{z}$ occurs twice if and only if it occurs in Legendre symbols of both forms (4.18) and (4.19), i.e., there are $i, j, h$ with $1 \leq i, j \leq l$,

$$
r+d_{i}=a_{z}, \quad k \nmid\left(r+d_{i}\right)
$$

and

$$
r+d_{j}-h=a_{z}, \quad k \mid\left(r+d_{j}\right), \quad 1 \leq h \leq k-1 .
$$

Whenever this is the case, i.e., the factor $u k+a_{z}$ occurs twice, we drop the factor $\left(u k+a_{z}\right)^{2}$ in the "numerator" of the Legendre symbol in question. Doing this with all the factors occurring twice, we finally arrive at a representation of the form

$$
e_{u k+r+d_{1}} e_{u k+r+d_{2}} \ldots e_{u k+r+d_{l}}=\left(\frac{g(u)}{p}\right)
$$

where either

$$
g(u)=1
$$

or $g(u)$ is a product of linear polynomials of the form $u k+b_{i}$ distinct modulo $p$ :

$$
g(u)=\left(u k+b_{1}\right)\left(u k+b_{2}\right) \ldots\left(u k+b_{y}\right)
$$

where

$$
b_{i} \not \equiv b_{j}(\bmod p) \quad \text { for } 1 \leq i<j \leq y .
$$

Note that the degree of $g(u)$ is at most the sum of the degrees of the polynomials in the "numerators" of the Legendre symbols corresponding to the numbers $e_{u k+r+d_{i}}$ in the sense (4.18) and (4.19). Since the degree of each of these polynomials is at most $k$, and $i$ may assume $l$ distinct values, the degree of $g(u)$ is

$$
\operatorname{deg} g(u) \leq k l .
$$

Now we show that it follows from the assumption $k \nmid l$ that case (4.23) cannot occur. We argue by contradiction: assume that (4.23) holds, i.e., each $u k+a_{z}$ mentioned above occurs twice so that each $a_{z}$ can be represented in both forms (4.20) and (4.21). Fix a quadruple $i, j, h$ and $z$ satisfying (4.20) and (4.21). Then for all $h^{\prime}$ with $1 \leq h^{\prime} \leq k-1$, write $a_{z^{\prime}}=r+d_{j}-h^{\prime}$. For each of these numbers $a_{z^{\prime}}$ the factor $u k+a_{z^{\prime}}$ appears in the Legendre symbol corresponding to $e_{u k+r+d_{j}}$ in sense (4.19). Since by our indirect assumption each $u k+a_{z^{\prime}}$ occurs twice, each of the $a_{z^{\prime}}$ must also have a representation in the form (4.20) so that each of the numbers $d_{j}-h^{\prime}$ is a $d_{i}$, i.e., each of

$$
e_{u k+r+d_{j}-1}, \quad e_{u k+r+d_{j}-2}, \ldots, e_{u k+r+d_{j}-k+1}
$$

occurs in the product in (4.22). Thus dropping all these factors $u k+a_{z}$, $u k+a_{z^{\prime}}$ (the latter corresponding to the fixed $a_{z}$ ) means to eliminate the contribution $(=1)$ of the $k$ e's in (4.27). 
If not all the factors $u k+a_{z}$ have been dropped yet, then we may repeat this procedure again by dropping $k-1$ distinct factors $u k+a_{z}$ each occurring twice, and corresponding to the contribution of $k$ further factors $e_{u k+r+d_{i}}$ in (4.22). Repeating this procedure again and again, finally by our assumption (4.23) we drop all the factors $u k+a_{z}$. In each step we consider the contribution of $k$ further factors $e_{u k+r+d_{i}}$ in (4.22) so that the total number $l$ of these factors must be an integer multiple of $k$. But this contradicts our assumption $k \nmid l$ and this contradiction proves that (4.23) cannot hold so that $g(u)$ must be of the form (4.24).

By (4.17) and (4.22), $S(r)$ can be rewritten as

$$
S(r)=\sum_{0 \leq u \leq(M-r) / k}\left(\frac{g(u)}{p}\right)
$$

where $g(u)$ is of the form (4.24). Defining $\bar{k}$ again by (4.13), we may write this sum as

$$
S(r)=\left(\frac{k^{y}}{p}\right) \sum_{0 \leq u \leq(M-r) / k}\left(\frac{f(u)}{p}\right)
$$

with

$$
f(u)=\left(u+b_{1} \bar{k}\right)\left(u+b_{2} \bar{k}\right) \ldots\left(u+b_{y} \bar{k}\right)
$$

where

$$
b_{i} \bar{k} \not \equiv b_{j} \bar{k}(\bmod p) \quad \text { for } 1 \leq i<j \leq y
$$

by (4.25), and

$$
\operatorname{deg} f(u)=y=\operatorname{deg} g(u) \leq k l
$$

by (4.26). By (4.29) and (4.30), we may apply Lemma 1 to estimate the sum in (4.28). By (4.8) and (4.31) we get

$$
\begin{aligned}
|S(r)| & =\left|\sum_{0 \leq u \leq(M-r) / k}\left(\frac{f(u)}{p}\right)\right|<9 k l p^{1 / 2} \log p \\
& \leq 9 k l(2 N)^{1 / 2} \log (2 N)<27 k l N^{1 / 2} \log N \quad \text { for } r=1, \ldots, k .
\end{aligned}
$$

(4.15) follows from (4.16) and (4.32), and this completes the proof of Theorem 6 .

5. Remarks to earlier papers of ours. Finally, we would like to make two remarks concerning our earlier papers [CFMRS1] and [CFMRS2]. In these two papers we studied the pseudorandom properties of the Liouville function $\lambda(n)=(-1)^{\Omega(n)}$ (where $\Omega(n)$ denotes the number of prime factors of $n$ counted with multiplicity). Write $L_{N}=\{\lambda(1), \ldots, \lambda(N)\}$. In particular, in [CFMRS1] we showed that assuming the generalized Riemann hypothesis, we have

$$
W\left(L_{N}\right)<N^{5 / 6+\varepsilon}
$$


It has been pointed out to us by Dr. Louis Goubin (Bull. PTS) that if one replaces the second half of our Lemma 1 there by a reference to a more recent result of Baker and Harman $[\mathrm{BH}]$, the exponent $5 / 6$ in (5.1) can be improved to $3 / 4$. We would like to thank Dr. Goubin for this comment.

Secondly, in [CFMRS2] we studied the behaviour of the Liouville function over polynomials and, in particular, over quadratic polynomials. We have learned recently that I. Kátai [Kát] had also studied the $\lambda$ function over quadratic polynomials but his results are different from ours.

\section{References}

[BH] R. C. Baker and G. Harman, Exponential sums formed with the Möbius function, J. London Math. Soc. 43 (1991), 193-198.

[CFMRS1] J. Cassaigne, S. Ferenczi, C. Mauduit, J. Rivat and A. Sárközy, On finite pseudorandom binary sequences III: The Liouville function, I, Acta Arith. 87 (1999), 367-390.

[CFMRS2] - - - - - - 一, On finite pseudorandom binary sequences IV: The Liouville function, II, ibid. 95 (2000), 343-359.

[EF] P. Erdős and Z. Füredi, The greatest angle among $n$ points in the d-dimensional Euclidean space, in: Combinatorial Mathematics (Marseille-Luminy, 1981), North-Holland Math. Stud. 75, North-Holland, Amsterdam, 1983, 275-283.

[Kah] J.-P. Kahane, Some Random Series of Functions, 2nd ed., Cambridge Stud. Adv. Math. 5, Cambridge Univ. Press, Cambridge, 1985.

[Kát] I. Kátai, Research problems in number theory II, Ann. Univ. Sci. Budapest Sect. Comput. 16 (1996), 223-251.

[MSá] C. Mauduit and A. Sárközy, On finite pseudorandom binary sequences I: Measure of pseudorandomness, the Legendre symbol, Acta Arith. 82 (1997), $365-377$.

[MSp] J. Matoušek and J. Spencer, Discrepancy in arithmetic progressions, J. Amer. Math. Soc. 9 (1996), 195-204.

[Ro] K. F. Roth, Remark concerning integer sequences, Acta Arith. 9 (1964), $257-260$.

[We] A. Weil, Sur les courbes algébriques et les variétés qui s'en déduisent, Act. Sci. Ind. 1041, Hermann, Paris, 1948.

Institut de Mathématiques de Luminy

CNRS UPR 9016, FRUMAM

Université de la Méditerranée

163 av. de Luminy, Case 907

F-13288 Marseille Cedex 9, France

E-mail: cassaigne@iml.univ-mrs.fr mauduit@iml.univ-mrs.fr
Department of Algebra and Number Theory Eötvös Loránd University

Kecskeméti u. 10-12 H-1053 Budapest, Hungary E-mail: sarkozy@cs.elte.hu

Received on 20.10.2000

and in revised form on 18.9.2001 